\title{
IST DAS BÖHMISCHE SPIELZEUG TATSÄCHLICH BÖHMISCH?
}

\author{
Hana DVOŘÁKOVÁ \\ Ethnographisches Institut, Mährisches Landesmuseum \\ 60200 Brno, Kobližná 1, Tschechische Republik
}

Schlägt man jedwede repräsentative den Ausdrücken der Volkskunst in böhmischen Ländern gewidmete Publikation auf, findet man darin neben klassichen Vertretern dieses Genres, wie z. B. Hinterglasmalerei oder Keramik, immer auch das Spielzeug. Spielsachen aus dörflichem Milieu - Gegenstände mit ganz spezifischer Bestimmung - deren Sendung von jener des künstlerischen Artefaks diametral unterschiedlich war, wurden paradoxerweise ex post zu Vertretern der Volkskunst. Für seine unbestreitbaren künstlerischen Werte wurde und wird das Spielzeug in die Kategorie der Volkskunst einbezogen und als ästhetisches Phänomen präsentiert. Auf diese Weise reiht es sich zu Ausdrücken der „Außerkunst“, unter Produkten spezialisierter dörflicher oder städtischer Handwerke, die allgemein und bewußt in die Sphäre der Volkskunst eingegliedert werden (JEŘÁBEK, 1978: 407). Diese Prämisse gilt für seine Existenz erst auf der zweiten Ebene, wo das Spielzeug zum Objekt des Sammler- und Forscherinteresses wurde. Ästhetische Qualitäten wurden vor allem durch Zugehörige der Intelligenz hervorgehoben. Anfang der 90er Jahre des 19. Jahrhunderts widmete ihm z. B. die begeisterte Bewunderin der Volkskultur, die Malerin Zdenka Braunerová ihr Interesse, die sich dadurch in ihrem Schaffen inspirieren ließ. $\mathrm{Zu}$ den nicht zahlreichen, aber zielbewußten Sammlern gehörte in derselben Zeit der Schriftsteller Vilém Mrštík, der die Spielsachen in seinem Interieur als dekoratives Element benutzte. 35 gedrechselte Holzspielsachen aus seinem Nachlaß, die heute im Olmützer Museum aufbewahrt werden, stellen eine der wenigen chronologisch sowie geographisch abgegrenzten Kollektionen dar und erlauben uns einen Einblick in die Welt der Kinderspiele auf dem südmährischen Lande an der Wende des 19. und 20. Jahrhunderts. Ansichten auf über volkstümliche Spielzeug waren unterschiedlich - die Intellektuellen waren mit seiner einfachen Form als Höhepunkt der vollkommenen Gestaltung zufrieden. Die Handelskammer in Pilsen dagegen, die für die Kleinindustrie zuständig war, stellte sich in ihrem statistischen Bericht aus dem Jahre 1877 zu gedrechselten Spielsachen aus Skašov ausgesprochen negativ: „Die Form sowie die Farbharmonie der Skašover Spielsachen sind sehr schlecht und verderben den Geschmack der Kinder." Zehn Jahre später lautet ihre Verurteilung noch schärfer: „Skašover Spielsachen sind gemein“, d. h. einfach, primitiv (zit. HERCík 1951: 12). Die einseitige Bevorzugung der ästhetischen Funktion ist beim Spielzeug irreführend und erweckt Verlegenheit ebenso wie die 


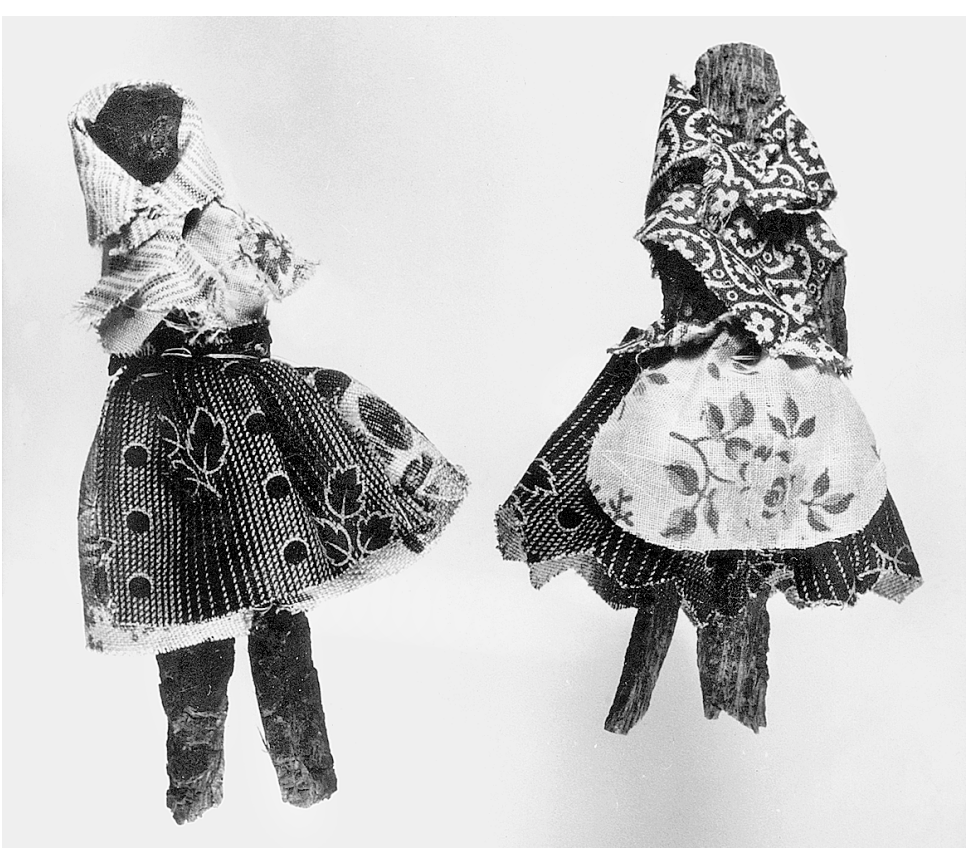

Fig. 1. Primitive Puppen aus der Rinde der Bäume, Mähren, Anf. des 20. Jh.

Wagheit des Begriffes selbst „Volksspielzeug“, der in der tschechischen volkskundlichen Terminologie neben ähnlichen Begriffen wie Volksmöbel, Volkskeramik usw. vorkommt. Ähnlich wie bei der Volksmöbel oder -keramik verbirgt sich unter dieser Bezeichnung vor allem die Produktion der spezialisierten Hausindustrie. Ihre Erzeugnisse stehen auf einem Ende der Wertskala, während auf dem anderen z. B. kleine Steine erscheinen, aus welchen die Kinderphantasie für eine Weile ein Spielzeug machte.

Böhmische und mährische Museumssammlungen bieten ein verengtes und verzerrtes Bild von der Entwicklung des Volksspielzeugs. Das Sammelwesen, das sich in seinen Anfängen an keine wissenschaftlichen Erkenntnisse stützte, ließ das geläufige, durch Kinder oder deren nächste Umgebung hergestellte Spielzeug außer acht und konzentrierte sich auf das Zusammenbringen von Belegen der vorwiegend hölzernen Spielsachen, an zweiter Stelle dann der keramischen und randweise der Textilspielsachen. Im Mittelpunkt der Aufmerksamkeit stand die Produktion der hausgemachten gedrechselten Spielsachen aus den wichtigsten Produktionszentren wie z. B. Skašov, Příbram, Horácko. Die gelegentliche Herstellung, z. B. Teufel aus getrockneten Früchten für Sankt-Nikolaus-Märkte, blieb als nicht attraktiv am Rande der Aufmerksamkeit. Durch dieses Herangehen litt das Spielzeug vielleicht mehr als jedwedes andere Genre, denn hinsichtlich seiner kurzen Lebensdauer können die Lücken nicht mehr gefüllt werden. Primitive Spielsachen, wie z. B. Wassermühlen, Spielsachen aus lebendem Holz, Federn, Früchten usw., die durch den österreichi- 


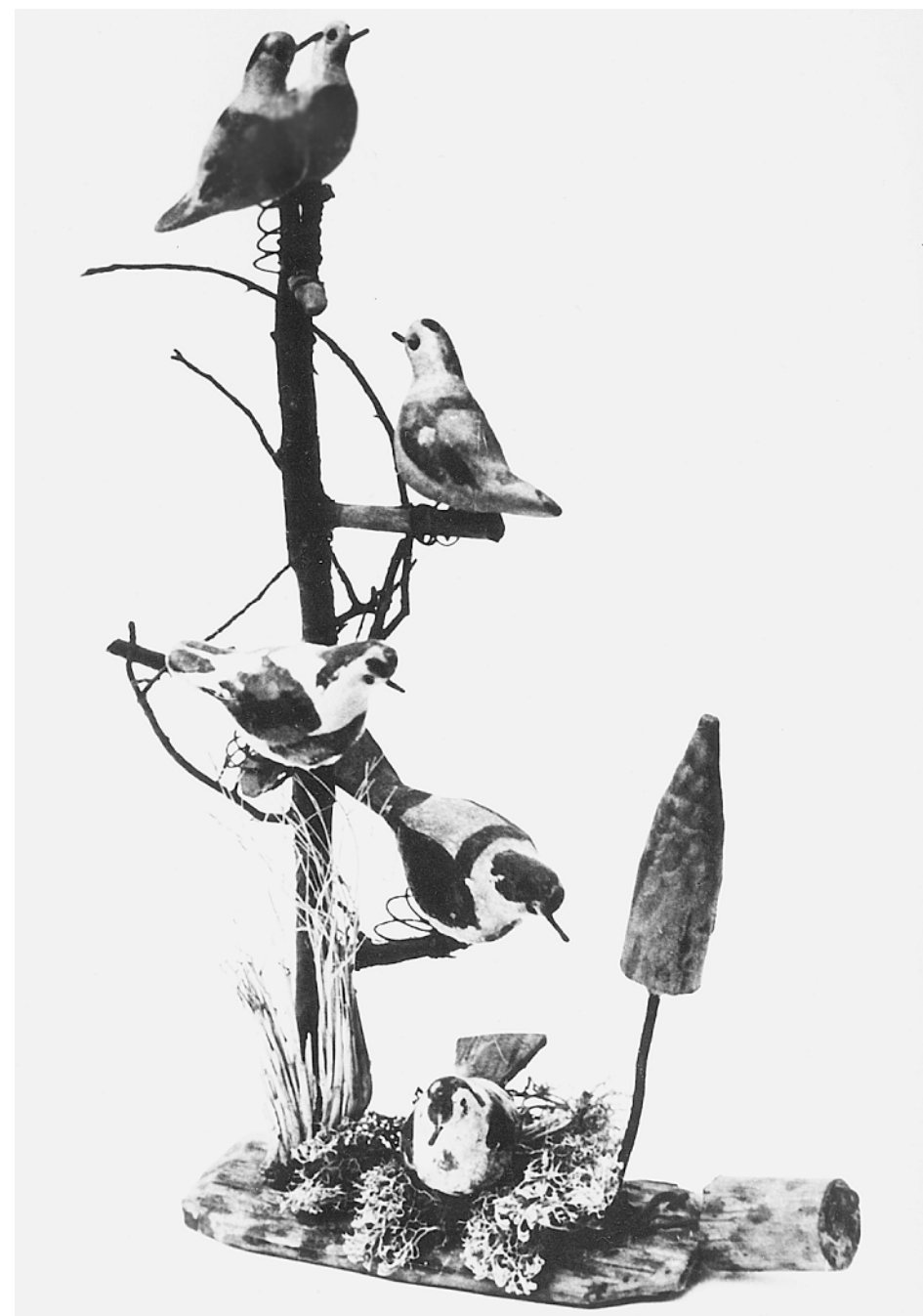

Fig. 2. Vögel aus getrocknetem Ton, Mähren, um J. 1920.

schen Forscher V. KUTSCHERA als „Bio-Spielsachen“, gelegentliches Spielzeug bezeichnet werden, kommen in (nicht nur) tschechischen Sammlungen sporadisch vor. Dies ist nicht durch den Mangel an Interesse für das allzu einfache, aus der ästhetischen Hinsicht uninteressante Spielzeug verursacht, da es in Museumsmagazinen fehlt. Die einmalige Benutzung gemeinsam mit dem aggressiven Milieu der Kinder waren die Ursache seines Verschwindens. Darin beruht der Vorteil des Spielzeugs im bürgerlichen und adeligen Milieu, wo wegen des hohen Preises viel mehr Belege erhalten blieben. Darüber hinaus können sie nach ikonographischen Quellen (z. B. Bildern) rekonstruiert werden. 
Das Interesse unserer Forscher und Sammler für das Spielzeug aus dem dörflichen und kleinstädtischen Milieu dauert, wenn auch in begrenztem Maße, ungefähr seit 100 Jahren an. Im Vergleich mit weiteren Bereichen der Volkskultur stand das Spielzeug einigermaßen abseits der Aufmerksamkeit. Die Zahl der veröffentlichten Studien oder Sammlungsgegenstände kann mit anderen Genres (z. B. Volksmöbel, Keramik) nicht verglichen werden. Museumsfonds beinhalten dann das im 19. und am Anfang des 20. Jahrhunderts hergestellte Spielzeug. Ältere Belege kommen sporadisch vor. Eine geschlossene Kollektion, die zu unseren ältesten gehört, bilden Spielsachen, die durch den Heimat-Museumsverein in Olmütz zusammengebracht wurden (127 Stücke). Die sich seit 1883 konstituierende Kollektion dokumentiert das Leben der Kinder in der Hannakei am Ende des 19. Jahrhunderts. Die Sammlung des Nationalmuseums in Prag entstand als Nachlaß der Tschecho-slawischen ethnographischen Ausstellung 1895 und nach jener von Olmütz gehört sie zu den ältesten bei uns. Ähnlich wie weitere verbinden diese beiden die Produktion böhmischer und mährischer Werkstätten mit Erzeugnissen berühmter Spielzeugzentren in der österreichischen Salzkammer, in Bayern und im Erzgebirge, die auf tschechischen Märkten, wo die ausländische Produktion mit einheimischem Angebot verschmolz, viel verkauft wurden. Dieses Durchdringen ist für das tschechische Milieu typisch und bildete ein spezifisches Klima heraus, in welchem sich das tschechische Spielzeug entwickelte. Zur Mischung im geographischen Sinne kommt noch das soziale Durchdringen hinzu, denn das Spielzeug aus städtischem Milieu gelangte als Geschenk oder weggelegte Sache aufs Land und umgekehrt - Bedienstete erzeugten Spielsachen für Kinder ihrer Herrschaften.

Die zahlreichsten materiellen und Archivbelege für das Fachstudium bietet die Hausproduktion hölzerner gedrechselter Spielsachen, die sich auf mehreren Stellen in eine Hausindustrie entwickelte. Sie zeigt eine breite Skala von Puppen, Pferdchen, Husaren, Möbeln, Musikinstrumenten, Vögeln, mechanischen Spielsachen, Pistolen usw., die aus dem traditionellen Rahmen der üblichen Produktion nicht ausweichen. Die Produktion konzentrierte sich in folgenden Ortschaften: Skašov bei Pilsen, ungefähr 10 Gemeinden in Ostböhmen (in der Umgebung von Hlinsko in Horácko), in Př́ibram und Králíky in Schlesien. Werkstätten in Kuttelberg der Hl. Katarina produzierten vor allem für deutsche Märkte kleine, aus gedrechselten Ringen gespaltete Figürchen (eine ähnliche Produktion findet man im deutschen Seiffen). Ähnlich wie in anderen Ländern wurde die Spielzeugproduktion durch die Wirtschaftskrise, d. h. den Verfall der Hauptbeschäftigung (vor allem Bergbau und Textil-Hausproduktion) und die Suche nach einem Ersatzlebensunterhalt in Wintermonaten angeregt. Ungünstige ökonomische Verhältnisse gemeinsam mit Naturbedingungen bildeten also in Mitteleuropa unabhänging voneinander die gleiche Basis, auf welcher sich die Hausproduktion in der Umgebung von Salzburg, in Bayern, auf der deutschen und tschechischen Seite von Erzgebirge sowie in böhmischen Zentren entwickelte. Schriftliche Quellen belegen die Produktion in umseren Zentren erst seit dem ersten Drittel des 19. Jahrhunderts. Es handelt sich also um eine relativ junge Tradition, die sich im Kontext mit dem Geschehen in den Nachbarländern entwickelte. Für die letztgenannten war Böhmen zunächst nur ein Ab- 


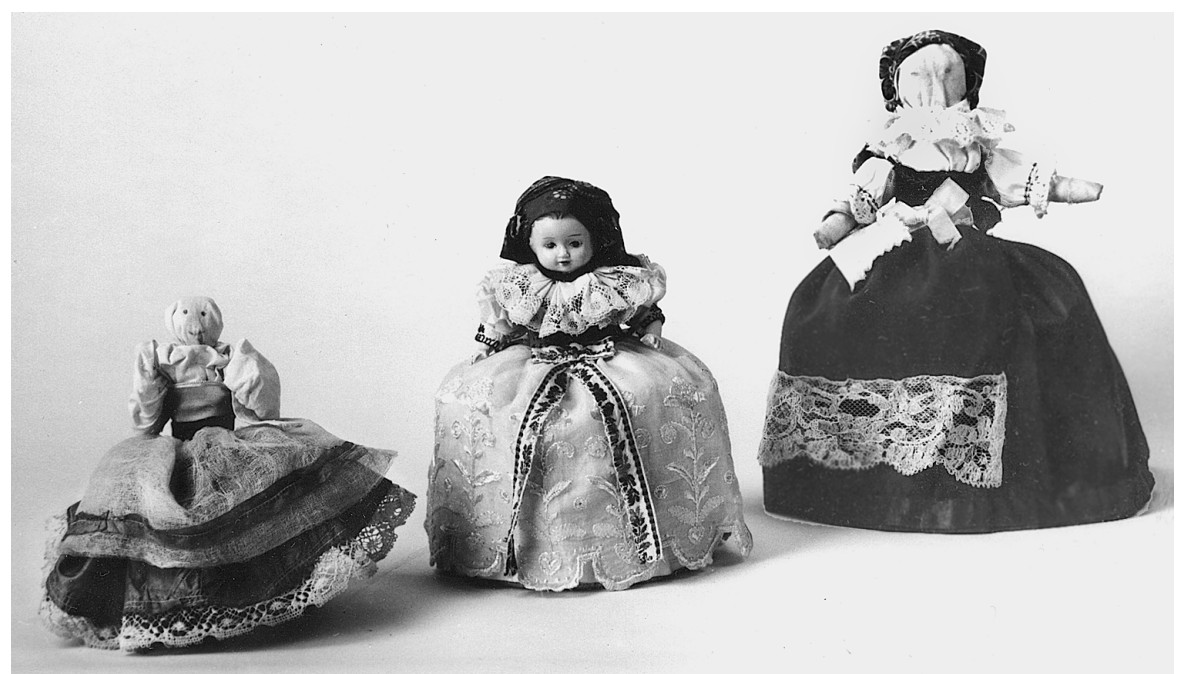

Fig. 3. Textilpuppen in Volkstracht, Mittelmähren und Umgebung Brünns, Ende 19. Jh. und ca. J. 1930.

satzgebiet. In österreichischen und deutschen Zentren war die Manufakturproduktion von Spielsachen bereits seit dem 16. Jahrhundert fest verankert und mittels eines gut organisierten Handelsnetzes mit Umladeplätzen und Lagern reichte sie bis auf unsere Märkte hin. Mit dem Vertrieb beschäftigten sich Nürnberger Handelsfirmen, die den Handel mit dem Spielzeug aus der Umgebung von Salzburg und aus Bayern vermittelten. Auf eingeführten Handelswegen lagen böhmische und mährische Städte, in deren Hinterland sich die Produktion gedrechselter Spielsachen entwickelte. Interessant ist die Information über Handelskontakte der Spielzeugfirma Oedel aus dem österreichischen Hallein mit dem germanisierten Iglau (Jihlava, eine Stadt mit entwickelter Textilproduktion und Erzabbau im Mittelalter). Das natürliche Hinterland Iglaus bildeten Weber aus Horácko, die sich nach dem Niedergang der Hausweberei der Spielzeugproduktion zu widmen begannen. In diesem Zusammenhang bieten sich Hypothesen über gegenseitige Einflüsse, die durch das Studium von Quellen in österreichischen Archiven erklärt werden könnten. Vergleicht man die tschechische Produktion mit der ausländischen, dann sind gewisse Übereinstimmungen in Produktonstypen sowohl mit Sonneberg in Thüringen als auch mit Berchtesgaden und Oberammergau in Bayern sowie mit dem sächsischen Spielzeug aus dem Erzgebirge zu beobachten (HERAIN 1953: 248). Von gegenseitigen Kontakten zeugt die Tatsache, daß tschechische Produkte zu Ende des 19. Jahrhunderts auch auf österreichischen und ungarischen Märkten zum Vorschein kamen. Einen der Belege von Handelskontakten stellt die Existenz eines speziellen Puppentyps dar, der als „Tirolerin“ bezeichnet wird, bei welchem die Farbausführung der Kleidung dem Geschmack der österreichischen Kunden entsprach. Der österreichische Forscher Erich URBANEK führt ausdrücklich an, daß der typische tschechische „Stelzenvogel" in Hallein und Berchtesgaden hergestellt wurde (URBANEK 1987: 73). Es gab 


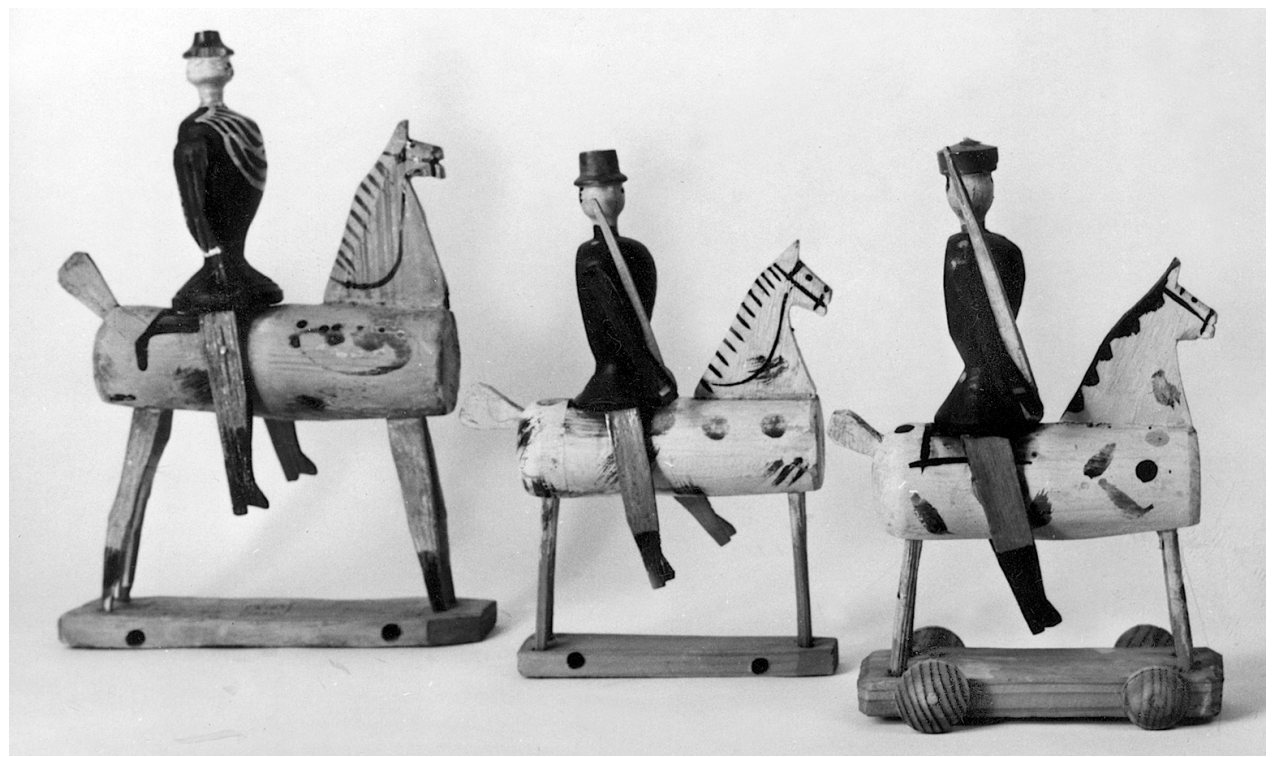

Fig. 4. Gedrechseltes Holzspielzeug, Gebiet Horácko, West Mähren, Anfang 20. Jh.

hier also einen starken Markt und das tschechische Spielzeug entwickelte sich in seinem Kontext. Die ähnliche Form is durch die Art und Weise der Bearbeitung und die gültigen Muster gegeben, an welchen sich die Hersteller halten mußten, falls sie in der Konkurrenz mit den schon eingeführten Waren bestehen wollten. Bei dem Vergleich einiger konkreter Erzeugnisse aus verschiedenen Produktionszentren sind gegenseitige Bindungen mehr als deutlich. Von der Verknüpfung am Anfang des 20. Jahhunderts zeugt die Tatsache, daß Halbprodukte mährischer Spielsachen zur endgültiger Formgebung nach Österreich geliefert wurden, woher sie als ein schon „Tiroler“ Erzeugnis zurückkamen: „Es gibt in Mähren gewerbetreibende Drechsler oder Schnitzer, die für den Wiener Markt verschiedene gedrechselte Spielsachen herstellen, die in Werkstätten der Verkäufer entweder durch verschiedene Abdruckbilder verziert oder direkt bemalt, ausgebrannt usw. werden und dann mit dem Anstrich der Tiroler und niederösterreichischen Industrie zurückkehren (SVAZ 1900: 57).

Die vier tschechischen Zentren der Produktion gedrechselter Spielsachen können in zwei Gruppen geteilt werden: in die erste gehört Príbram und Králíky, die durch das gleiche soziale Hinterland verknüpft sind, nämlich die Beschäftigung im Bergbau (ähnlich wie im Erzgebirge oder in Berchtesgaden hing Spielzeugproduktion mit dem Verfall des Bergbaus zusammen). In den beiden Fällen handelt es sich auch um bedeutende Wallfahrtsorte, was in Př́bram das Sortiment beeinflußte, denn es kamen dort Spielsachen in Form von Nonnen und Kranzjungfern vor, die die Bahre mit der Wunderstatue trugen. Zu einer Ergänzung wurde die Herstellung von Weihnachtskrippen und Devotionalien. Auch die angewandte Technik, d. h. der 


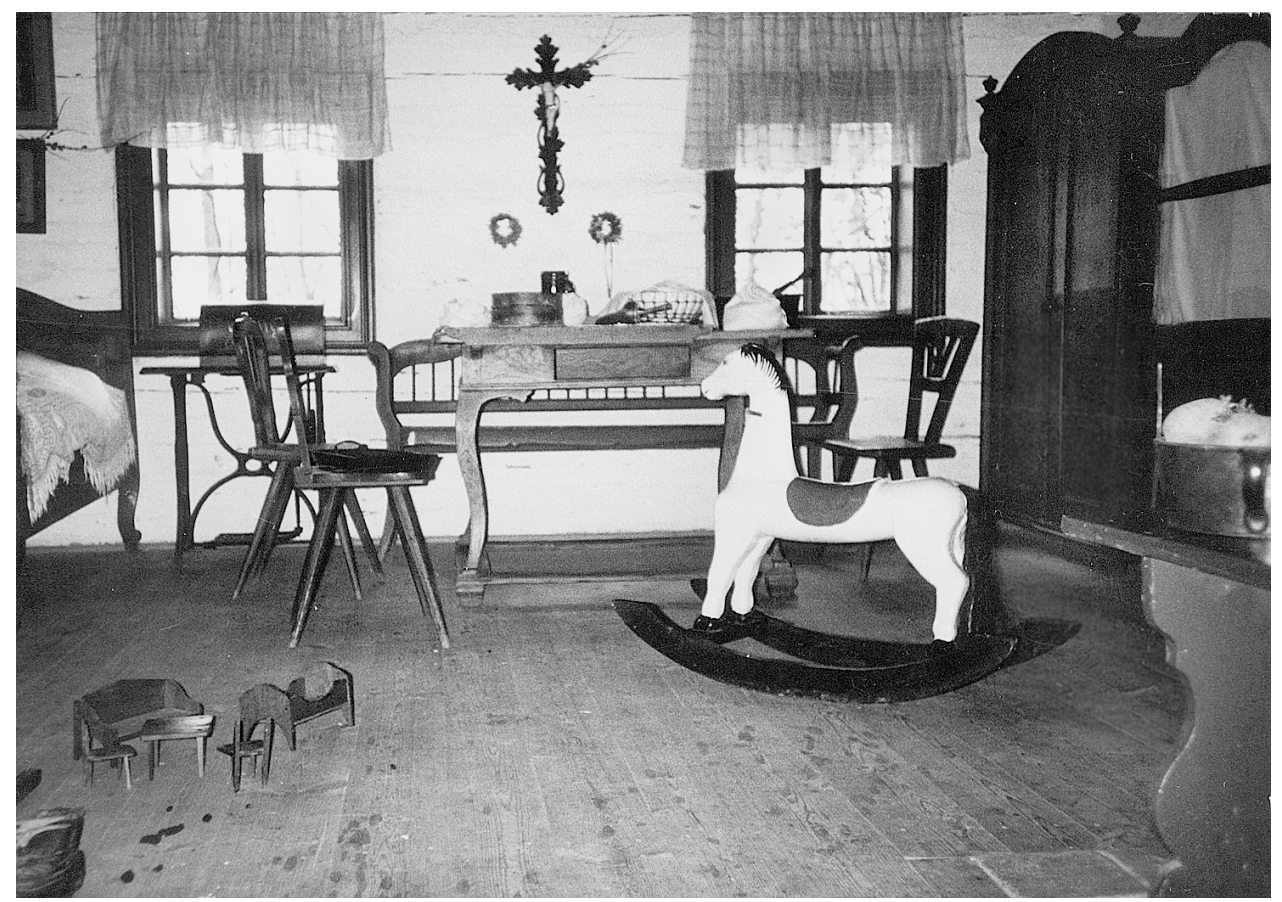

Fig. 5. Rekonstruktion der Bauernstube aus dem Gebiet Horácko mit den Spielzeugen. Freilichtmuseum Veselý kopec bei Hlinsko, Ost Böhmen

gedrechselte Korpus mit Brotmasse modelliert, war in den beiden Ortschaften gleich. Interessant ist auch die Zusammensetzung der tschechisch und deutsch sprechenden Bevölkerung. Eine gegenseitige Verbindung ist jedoch nur hypothetisch und konnte bisher nicht bewiesen werden. Während in den beiden oben erwähnten Zentren die Spielzeugproduktion mit der Herstellung von Weihnachtskrippen verbunden ist, wird weder in Skašov noch in Horácko eine ähnliche Verbindung erwähnt. Diese Tatsache kommt deutlich auf dem Gebiet von Horácko zum Ausdruck, wo die Spielzeug- und Weihnachtskrippenproduktion nebeneinander existierte, aber nie gemeinsam auf einer Stelle. In der Umgebung von Hlinsko gehörte die Spielzeugproduktion zu den Hauptbereichen der holzbearbeitenden Industrie. Die Blütezeit der Hausproduktion von Spielsachen fällt in die zweite Hälfte des 19. Jahrhunderts.

Der Rückgang der Hausproduktion zu Anfang des 20. Jahrhunderts wurde durch die billigere fabrikmäßige Serienproduktion und die Veränderung der Nachfrage verursacht. Eine weitere Entfaltung sollte durch Ausstellungen (SVAZ 1913; 35) sowie Kurse zur Förderung der Gewerbe unterstützt werden, die durch den Staat, das Gewerbeamt und Interessengemeinschaften organisert wurden. In dem ersten davon, der 1907 in Skašov stattfand, wurden die Spielzeughersteller mit einer Neuigkeit bekanntgemacht, nämlich mit Anilinfarben, deren Verwendung jedoch 


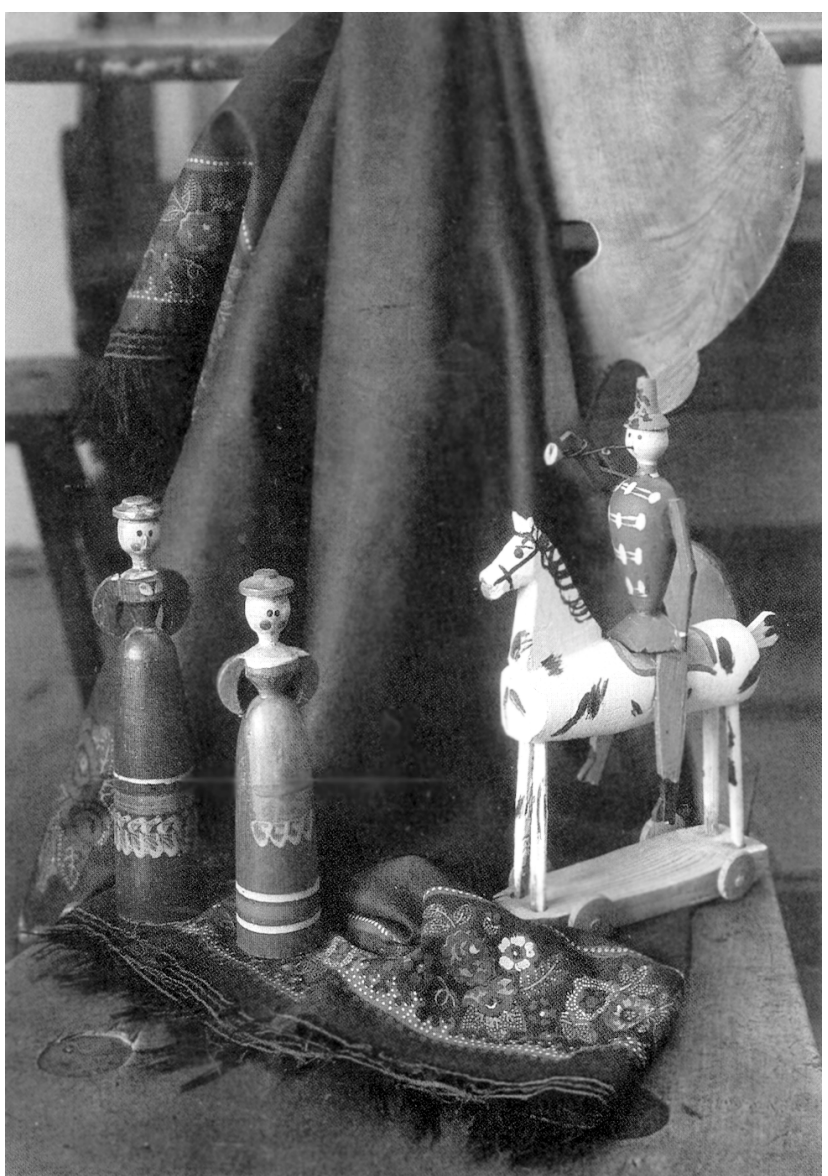

Fig. 6. Kollektion gedrechselter Holzspielzeuge, Gebiet Horácko, West Mähren, Anf. 20. Jh.

eine Verschlechterung der Farbigkeit und des Aussehens brachte. Die Hausproduktion ging zugrunde, die überlebenden Hersteller verkauften ihre Waren nur auf Märkten und in Wallfahrtsorten. Die Expansion der entstehenden Industrie trug zum Untergang eines der traditionellen Bereiche der Volksproduktion bei.

Anfang des 20. Jahrhunderts kamen die Bemühungen zu Tage, der Entwicklung des Spielzeugs die erwünschte Richtung zu geben, und zwar seitens der offiziellen künstlerischen Kreise, die sich damit zu beschäftigen begannen. In Hořice, im Vorland des Riesengebirges, gründete Prof. Kysela eine künstlerische Genossenschaft, in welcher er versuchte, die zurückgehende örtliche Produktion von flachen Spielsachen zu fördern. Die enge Zusammenarbeit der Hořicer Genossenschaft mit vornehmen zeitgenössichen Künstlern war ganz vereinzelt. Es handelte sich praktisch um den Anfang der Zusammenarbeit des Designeurs mit dem Hersteller. Die Hořicer Spielsachen gingen von traditionellen Techniken heraus, wobei die Verzie- 


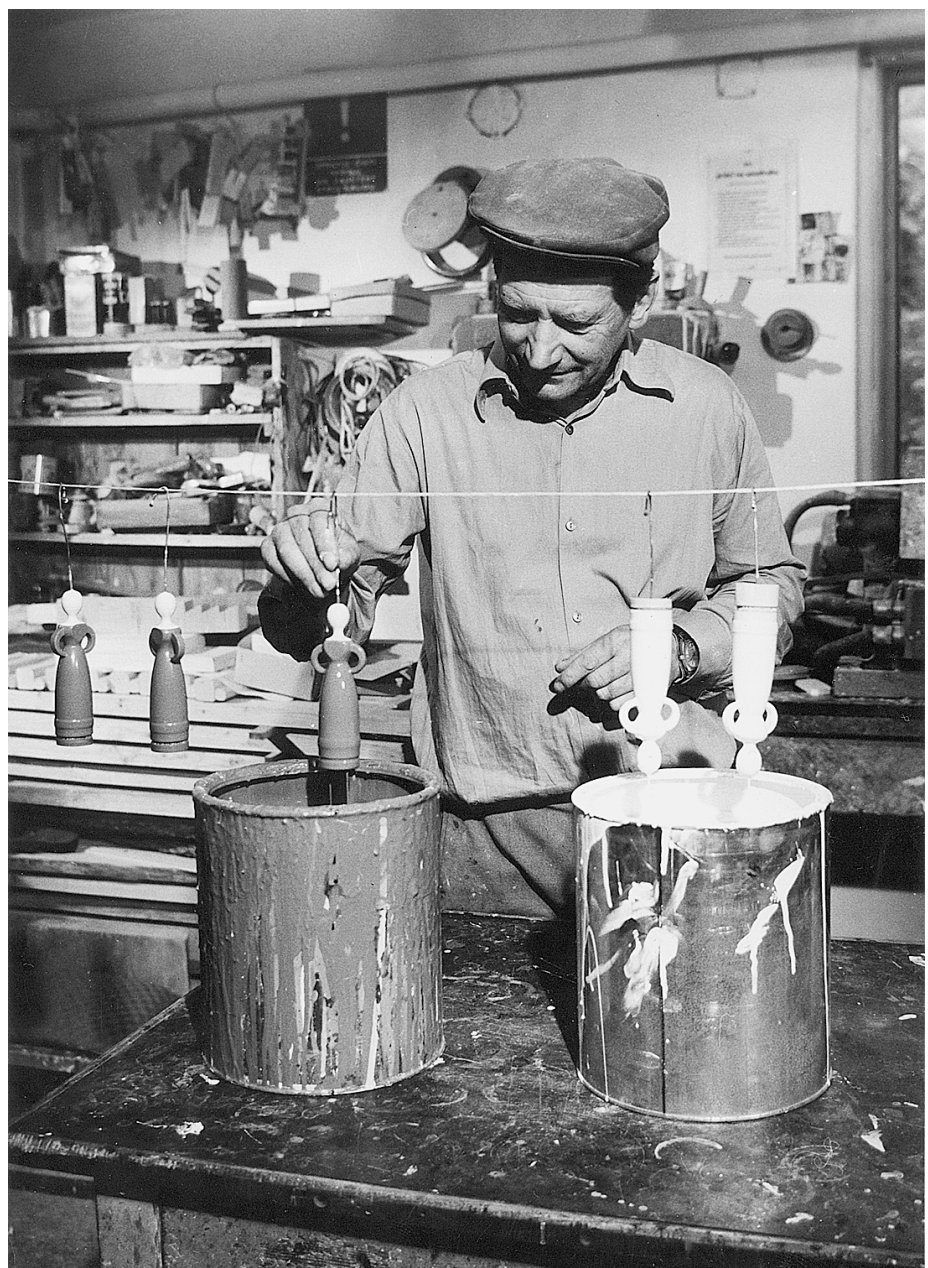

Fig. 7. Der Hersteller gedrechselter Holzspielzeuge Zdeněk Bukáček aus dem Dorf Krouna bei Arbeit. Krouna, J. 1983.

rung dem Dekor im sog. „Nationalgeist“ entsprach. Es kamen dort Figürchen in Tracht sowie Bauernmöbel mit „Volksornament“ verziert vor. Die Genossenschaft hatte kein langes Leben - sie unterlag dem kommerziellen Druck und dem Mangel an Interesse seitens der Öffentlichkeit. Die Gründungsmitglieder gingen nach 1918 in die neu konstituierte künstlerische Genossenschaft Artěl (1908-1934) über, aber auch dort waren erfahrene Künstler nicht imstande, das Programm der Herstellung des qualitätsvollen Spielzeugs zu realisieren und die Belebung der traditionellen Spielzeugproduktion auf dem Lande (Zusammenarbeit mit Spielzeugmacher in Skašov) zu erzielen. Es stellt sich die Frage, welche Faktoren ihren Niedergang verursachten: der erste Weltkrieg, das vorzeitige Ableben der Gründer oder die 


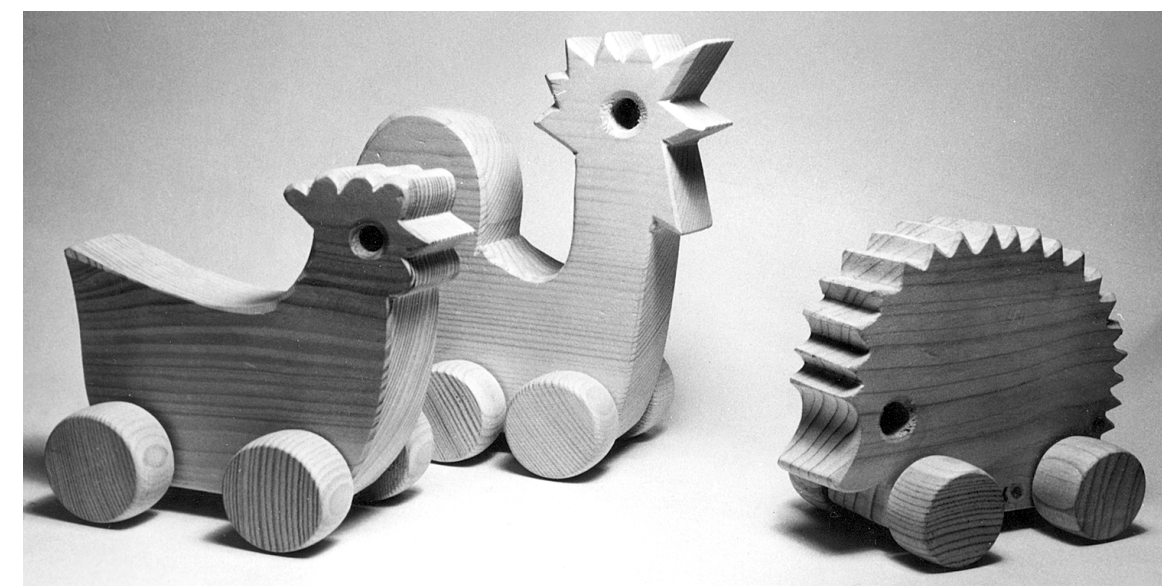

Fig. 8. Holzspielzeuge aus der Produktionszentrale für volkstümliches Kunstgewerbe, ca. J. 1980.

schlechte Schätzung der Situation auf dem Markt. Es ist die Tatsache, daß das Atelier-Spielzeug, von dem traditionellen Spielzeug herausgehend, als ein Ziergegenstand wahrgenommen wurde. Zur Zeit des ersten Weltkriegs hielt die Hausproduktion von Spielsachen praktisch auf und wurde nicht einmal nach 1918 erneuert. $\mathrm{Zu}$ einer bedingten Entwicklung kam es in der Periode des zweiten Weltkrieges, wo eine Konjuktur auftrat. Die Spielzeugproduktion der kleinen Werkstätten sollte Lücken auf dem Markt ausfüllen. Das Ergebnis war ein starker Verfall der Qualität, der die Degeneration der Spielzeugproduktion als Ganzes zu Folge hatte. Schuld war daran auch die Einführung neuer Technologien (elektrische Drechselbänke, Spritzantrag von Azetonfarben).

In den 50er Jahren beginnt auf der Basis des Staatsunternehmens eine weitere Etappe der tschechischen Spielzeugproduktion. Der Nationalbetrieb Tofa einbeschloß nicht nur die größte Firma Schowanek aus Albrechtice, sondern auch kleine Spielzeugwerkstätten. Im Jahre 1950 wurde ein Beirat für Spielzeug als ein gemeinsames Organ der Produktion und des Handels konstituiert, der die ganze Produktion von der pädagogischen, ideologischen, ästhetischen und Produktionsseite her einzuschätzen hatte. Selbständige Hersteller, die in Intentionen des traditionellen Spielzeugs schufen, wurden zu Angestellten der staatlichen Institution „Ústředí lidové umělecké výroby“ (ÚLUV - Zentrale für volkstümliches Kunstgewerbe), deren Produktion sich auf den Grundlagen der traditionellen Volkskultur entfaltete. Künstler, Ethnographen und Hersteller versuchten, einige Typen des traditionellen Spielzeugs zu rekonstruieren und auf dieser Basis neue Typen zu schaffen. Das Ergebnis war ein handwerklich perfektes, künstlerisch wertvolles, geschmackvolles und gefahrloses Spielzeug aus qualitätsvollem Material. Die finanziell und programmhaft anspruchsvolle Wiederbelebung des Volksspielzeugs mittels ÚLUV unter der Aufsicht von Künstlern und Ethnographen konnte nur dank der Unterstützung seitens des Staates erfolgen. 
Neben der ästhetischen Funktion wurde (und wird auch heute) dem Spielzeug auch die Funktion des offiziellen Nationalrepräsentanten zugeschrieben. Dieser Prozeß wurzelt in der nationalen Wiedergeburt im 19. Jahrhundert. Mit dem immer stärkeren Bewußtwerden eigener Existenz suchte auch die tschechische Gesellschaft den Weg, sich von dem sie umgebenden deutschen Element zu unterscheiden und sich als ein selbständiges Subjekt mit eigener Geschichte durchzusetzen. Dies geschah mittels der Belege der Dorfkultur, die auf diese Weise auf die Ebene der Nationalkultur erhoben wurde. Im Geiste zeitgenössischer Ansichten bot die Volkskultur eine fast unerschöpfbare Quelle von Beispielen, die das nationale Eigenleben belegten. $\mathrm{Zu}$ offiziellen Repräsentanten der Nation wurde auch das Volksspielzeug gereiht, und zwar in zwei Formen: Die ersten stellten logisch Puppen in einer Kleidung dar, die eine Miniatur der traditionellen Volkstracht war. Gemeinsam mit Volksstickereien brachte sie Zdenka Braunerová in den Jahren 1890-1891 nach Paris, wo sie zum Bestandteil eines die tschechische Kultur vorstellenden Basars wurden: „Spielzeuge aus der mährischen Slowakei stehen in Paris bei A. France, Huysmanns, E. Zola“. Die Funktion der Nationalrepräsentation behielten die Trachtenpuppen auch im Verlauf der kommenden Jahre. Auch heute sind die beliebten Trachtenpuppen nicht nur ein verbreitetes Touristensouvenir, sondern neben Gegenständen aus geschliffenem Glas auch ein Repräsentationsgeschenk (siehe z. B. Trachtenpuppen, die die Stadt Brünn ihren Partnerstädten schickt). Im Fall der Trachtenpuppen wird es wohl richtig sein, sie für eine gewisse Analogie des historischen Puppe-Mannequins zu halten, das mit zeitgenössischen Modetrends bekannt macht.

$\mathrm{Zu}$ einem Nationalsymbol wurde auch das gedrechselte Holzspielzeug mit farbiger Oberfläche, das vor allem als Puppe, Pferdchen oder Husar auf Pferd vorkommt. Wie es Photographien belegen, konnte sie auch unsere bisher größte ethnographische Veranstaltung - die Tschecho-slawische ethnographische Ausstellung 1895 - nicht entbehren. Dies ist desto paradoxer, daß gerade dieses Genre die meisten übereinstimmenden Merkmale mit Erzeugnissen aus deutschen und österreichischen Zentren aufweist. Diese Spielsachen wurden für die breite Öffentlichkeit zum Symbol des tschechischen Spielzeugs überhaupt.

Der gegenwärtige Aufschwung des Tourismus führt zum zahlenmäßigen Wachstum der Hersteller und zur Herausbildung des Spielzeugmarktes. Das in Intentionen der traditionellen Produktion hergestellte Spielzeug überlebte den Untergang des vom Staat subventionierten ÚLUV und in den Bedingungen des Privatunternehmens kam es paradoxerweise zu seiner Entfaltung. Es kommt gemeinsam mit dem Atelier-Spielzeug vor, mit welchem es durch das gleiche Material verbunden ist d. h. hauptsächlich ungefärbtes Holz, Drechseltechnik und einfache Form. Solche Gegenstände sind vor allem als Souvenir gemeint. So geriet eines der billigsten Erzeugnisse der ehemaligen Hausproduktion in die Rolle des teueren Erinnerungsgegenstandes. Es ist bemerkenswert, da auch am Ende des zweiten Jahrtausends Artefakte der längst erloschenen Dorfkultur als ein Kulturindikator einwirken und zur Sujetquelle zur Herausbildung des Bildes der nationalen Spezifizität geworden sind. Diese Situation ist noch interessanter, wenn man sich der Tatsache bewußt wird, daß 
die Massenproduktion der dritten Welt europäische Märkte mit Kopien des traditionellen Holzspielzeugs aus dem Erzgebirge mit der Etikette „Made in China“ überschwemmt. Ist es die Frage der Zeit, der Integration oder der Modenachfrage, wann diese Etiketten auch auf dem Symbol der tschechischen Tradition - dem Volksspielzeug erscheinen?

\section{LITERATUR}

BACHMANN, M.-LANGER, R.

1957: Berchtesgadener Volkskunst. Leipzig.

BRAND, Josef

1989: Z minulosti domácké výroby skašovských hraček. Preštice.

FIXL, Vladimír

1976: Půlstoletí české hračky. In: Umění a řemesla, 1976, 4, $20-23$.

HERAIN, Karel

1953: Nase lidové hračky. In: Věci a lidé, 4, 1953, 236-250.

HERCIK, Emanuel

1951: Československá lidová hračka. Praha 1951.

JEŘABEK, Richard

1978: Lidové umění a vytvarná kultura lidu. In: Slovenský národopis, 3, 1978, 405-412.

JOHNOVA, Helena

1978: Horácké lidové hračky. Katalog. Nová město n. Moravě.

KUTSCHERA, Volker

1983: Spielzeugmuseum (Sammlung Folk) Salzburg. Salzburg.

LINDNER, Simon

1979: Das Sonneberger Spielzeugmuseumsbuch. Leipzig.

URBANEK, Erich

1987: Berchtesgaden. In: Schallaburg 1987, 80-83. Schallaburg.

1987: Die kaiserlich königlich privilegierte Holzwaren-Fabrik Compagnie in Hallein. In: Schallaburg (1987: 70-77); Schallaburg.

SVAZ

1913: III., 4, 57-60.

TVAR

1952: 10 